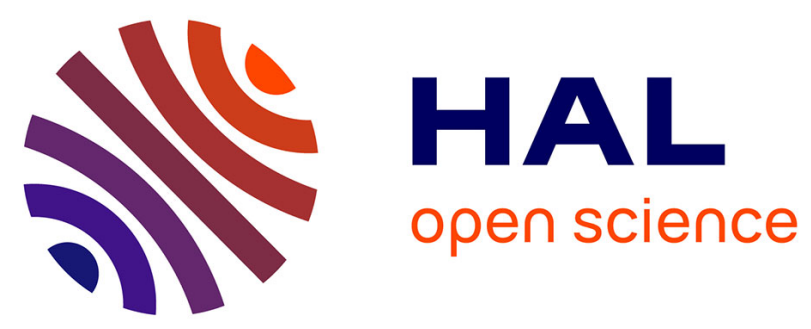

\title{
TP53 and 53BP1 Reunited
}

Thierry Soussi, Guido Kroemer

\section{To cite this version:}

Thierry Soussi, Guido Kroemer. TP53 and 53BP1 Reunited. Trends in Cell Biology, 2017, 27 (5), pp.311-313. 10.1016/j.tcb.2016.10.004 . hal-02318165

\section{HAL Id: hal-02318165 \\ https://hal.sorbonne-universite.fr/hal-02318165}

Submitted on 16 Oct 2019

HAL is a multi-disciplinary open access archive for the deposit and dissemination of scientific research documents, whether they are published or not. The documents may come from teaching and research institutions in France or abroad, or from public or private research centers.
L'archive ouverte pluridisciplinaire HAL, est destinée au dépôt et à la diffusion de documents scientifiques de niveau recherche, publiés ou non, émanant des établissements d'enseignement et de recherche français ou étrangers, des laboratoires publics ou privés. 
TP53 and 53BP1: reunited

T Soussi $i^{1-4^{*}}$

G Kroemer2-8

${ }^{1}$ Department of Oncology-Pathology, Karolinska Institutet, Cancer Center Karolinska (CCK) R8:04, Stockholm SE-171 76, Sweden

2 Université Pierre et Marie Curie, Paris, France;

3 INSERM U1138, Centre de Recherche des Cordeliers, Paris, France;

${ }^{4}$ Equipe11 labellisée Ligue Nationale contre le Cancer, Centre de Recherche des Cordeliers, Paris, France;

5 Université Paris Descartes, Paris, France;

${ }^{6}$ Metabolomics and Cell Biology Platforms, Institut Gustave Roussy, Villejuif, France;

7 Pôle de Biologie, Hôpital Européen Georges Pompidou, AP-HP, Paris, France

${ }^{8}$ Karolinska Institute, Department of Women's and Children's Health, Karolinska University Hospital, Stockholm, Sweden

*Correspondance: thierry.soussi@ki.se

Keywords: 53BP1; TP53; Cell cycle; Checkpoints 
According to the Uniprot database, 990 proteins physically interact with TP53 (http://www.uniprot.org/uniprot/P04637). It is currently unknown which among these interactions are biologically meaningful, a question that is difficult to assess, as such interactions could be restricted to a specific context and/or a particular cell type. Nonetheless, it can be expected that many if not most of these reported interactions will not withstand detailed exploration and hence turn out to be artefacts. In 1994, Iwabuchi et al. identified two unrelated cellular proteins, 53BP1 and 53BP2, as specific interactors with TP53 [1]. 53BP2, renamed ASPP1 for Apoptosis-stimulating of p53 protein 1, has been shown to be a member a large multigene family that includes ASPP1, ASPP2, and iASPP [2]. ASPP1 and 2 regulate TP53 by enhancing the in vivo DNA binding and transactivation function of TP53 on promoters of proapoptotic genes, whereas iASPP has the opposite effect. Although this function of 53BP2/ASPP1 is closely linked to TP53, 53BP1 rapidly left the world of TP53 to join that of DNA damage response pathways (DDR) as a key scaffold protein recruited to damage chromatin after DNA double-strand breaks (DSBs) [3,4]. Eukaryotic cells use two major pathways to repair DSBs: homologous recombination (HR) and non-homologous end joining (NHEJ). NHEJ directly ligates broken DNA ends together without requiring extended homologies. NHEJ is therefore error-prone and although such errors may be potentially detrimental, they can contribute to the generation of immune diversity in B and T lymphoid cells. 
On the other hand, in the case of $H R$ in $S$ or $G 2$, the cell requires a sister chromatid template as a source for any lost genetic information created by the DNA damage. Because HR uses such template as source of genetic information, this process is considered to be essentially error-free. Two novel pathways, single-strand annealing (SSA) and alternative end-joining (alt-EJ) have been identified in recent years, both requiring DNA end-resection and are therefore both error-prone.

P53BP1 is one of the factors that regulates this choice between the various DNA repair modalities by promoting NEHJ and repressing HR. It is noteworthy that recent studies have shown that 53BP1 also has a non-passive role in late S and G2 and promotes genetic stability by preventing hyper-DNA resection associated with SSA and promoting error-free HR. None of these activities requires an interaction with TP53 [4].

Four recent papers have brought 53BP1 back into the field of TP53 [58]. Three groups used a genome-wide screen to identify novel elements associated with the centrosome surveillance pathway in the human retinal pigmented epithelial hTERT-RPE1 cell line $[5,7,8]$. Following centrosome loss, this cell line exhibits irreversible G1 arrest that requires both TP53 and P21. The three groups identified 53BP1 as well as the deubiquitinase USP28 as two essential components acting upstream of TP53, as deletion of either of these components impairs induction of TP53 and G1 arrest after centrosome depletion. USP28 is able to deubiquitinate TP53 in vitro and a mutant devoid of deubiquitinase activity failed to stabilize TP53 in vivo $[6,7]$. 
Interestingly, TP53 and USP28 are recruited to 53BP1 via distinct, but proximal, regions of the BRCT domain, suggesting that the presence of these two proteins in close contact is essential for this cellular response [6].

This pathway is highly specific to centrosome losses, as TP53 accumulation and P21 induction after DNA damage induced by doxorubicin are not impaired in cells lacking either USP28 or 53BP1 .

Intriguingly, the function of 53BP1 associated with this centrosome surveillance pathway is totally distinct from its activity in the DDR. Cells expressing 53BP1 mutants with either point mutations or deletions of the BRCT domain are unable to arrest their cell cycle following centrosome loss, despite a normal DDR. On the other hand, 53BP1 mutants lacking the DDR function are still able to mediate centrosomal surveillance. The upstream stimulus that activates this pathway is currently unknown. The fourth paper directly addressed 53BP1 function in a panel of breast carcinoma MCF7 sublines that were partially or totally deficient for P53BP1 or TP53. In this system, loss of 53BP1 impairs several transcriptional programs of TP53 induced after irradiation or by the use of nutlin-3, a small molecule that antagonizes negative regulation of P53 by the ubiquitin ligase $\mathrm{mdm} 2$.

It is noteworthy that all four studies used cell lines that were genetically engineered to express normal levels of proteins that were mutated by means of the CRISPR-Cas9 methodology, hence avoiding the overexpression of exogenous genes via transfection. This technology may be expected to yield results that are closer to biological reality than previously used approaches. 
Returning to the origins of the discovery of 53BP1 and reinterpreting this interaction from the perspective of TP53 protein has revealed exciting findings. Although numerous proteins interact with TP53, most of these interactions involve the amino- or carboxy-termini of the protein, which are two flexible and accessible domains. In contrast, the interaction with 53BP1 occurs in the central core domain of the protein that includes the DNA binding region. Furthermore, a key observation reported by Iwabuchi et al. is that several oncogenic TP53 mutants found in human cancer are unable to interact with 53BP1 [9]. The specificity of the 53BP1-TP53 interaction was reinforced by analysis of the crystal structure established between the BRCT domain of 53BP1 and the core domain of TP53 (Figure 1). This interaction requires the $\mathrm{L} 2$ and $\mathrm{L} 3$ Loops as well as the $\mathrm{H} 1$ helix of TP53, including residues 248 and 273, two DNA-contact residues that are frequently mutated in human cancer $[10,11]$. Due to steric hindrance, it is likely that TP53 cannot engage 53BP1 and DNA binding simultaneously, suggesting that these two functions are independent from each other. It is noteworthy that loop L1, another region of TP53 essential for DNA recognition, is dispensable for the interaction between the two proteins (Figure 1).

Although the centrosome surveillance pathway requires an interaction between the BRCA1 $\mathrm{C}$ terminus (BRCT) domains of P53BP1 and TP53, previous studies have already shown that the Tudor motif of 53BP1 binds to dimethylated Lys 270 (K270me2) and 382(K382me2), localized in the carboxy-terminus of TP53. While the interaction with K270me2 is associated with transcriptional activation of TP53, the interaction with K382me2 recruits 
TP53 to damage DNA for an as yet unknown function. The ways in which the various activities of 53BP1 are coordinated during the cell cycle and the mechanisms that regulate their switch are still enigmatic.

The information provided by these four articles adds a new layer of complexity to the number of pathways regulated by TP53, and suggests that the docking activity of 53BP1 could act as a hub to distribute a spatiotemporally defined role to each molecular pathway involved in distinct types of stress.

Based on the discovery of the role of 53BP1 in keeping at bay centrosomeless cells via a specific interaction with TP53, several new burning questions arise. First, what is the contribution of the defect of TP53-53BP1 interaction in tumor cells expressing mutant TP53? Although we do know whether impaired DNA binding leads to loss of the transcriptional activity of TP53, it is possible that failed interactions between the two proteins could also contribute to the loss of function of TP53. The corollary of this observation is for example that a cancer-associated TP53 mutant in loop L1, which is essential for DNA binding but not involved in the interaction with 53PB1, should still be able to sustain G1 arrest after centrosome loss. If this prediction is correct, it will add yet another piece to the giant jigsaw puzzle composed by the heterogeneity of TP53 mutants. The TP53 gene expresses multiple isoforms truncated at the amino- and carboxy-termini, and both the DN40 and DN133 TP53 isoforms should be able to interact with 53BP1 (Figure 1) raising yet another set of interesting questions about the true target of 53BP1. 
Finally, and most importantly, the question comes up as to whether the TP5353BP1 interaction is relevant to cell transformation and through which mechanisms centrosome loss could contribute to cellular transformation. Although most research focuses on the relationship between genetic stability, centrosome amplification and hyperploidy in relation to cell transformation, it is perhaps time that we examine centrosome loss as an oncogenic mechanism. 
Figure 1

Crosstalk between TP53, 53BP1 and USP28. TP53 interaction with DNA requires correct folding of four highly conserved regions including loops $L 1$, L2, L3 and the LSH motif. DNA contact residues include notably amino acids 120, 248 and 273. The TP53 deltaN isoforms start at codon 40, 133 or 160 (red arrow heads). Dimethylated Lys 370 and 382 of TP53 (red dots) are recognized by the Tudor domain of 53BP1. The BRCT domain of 53BP1 requires only the $L 2, L 3$ and $L S H$ regions of the TP53 protein for the interaction. Mutation of amino acids 1845 or 1861 impairs this recognition, whereas mutations of amino-acids 1811 or 1814 (yellow dots), localized at the opposite surface of the BRCTs domain, impair the interaction with USP28. The domain of USP28 involved in binding to USP28 has not yet been identified, but mutations in the catalytic domains (blue dots) impair USP28 deubiquitinase activity and TP53 stabilization.

Abbreviations: BRCT, BRCA1 C-terminus; LSH, Loop Sheet Helix; UCH-1, ubiquitin carboxy-terminal hydrolases, Cys box); UCH-2, ubiquitin carboxyterminal hydrolases, His box); UDR, ubiquitin-dependent recruitment;

The 53BP1 protein is shown in reverse orientation (carboxy-terminus to amino-terminus) to emphasize the various interactions. Proteins are not drawn to scale. 
References

1 Iwabuchi, K., Li, B., Bartel, P. and Fields, S.

Use of the two-hybrid system to identify the domain of p53 involved in oligomerization.

Oncogene, 8 (1993), 1693-1696

2 Sullivan, A. and Lu, X.

ASPP: a new family of oncogenes and tumour suppressor genes.

Br J Cancer, 96 (2007), 196-200

3 Panier, S. and Boulton, S. J.

Double-strand break repair: 53BP1 comes into focus.

Nat Rev Mol Cell Biol, 15 (2014), 7-18

4 Zimmermann, M. and de Lange, T.

53BP1: pro choice in DNA repair.

Trends Cell Biol, 24 (2014), 108-117

5 Lambrus, B. G., Daggubati, V., Uetake, Y. et al.

A USP28-53BP1-p53-p21 signaling axis arrests growth after centrosome loss or prolonged mitosis.

J Cell Biol, 214 (2016), 143-153 
6 Cuella-Martin, R., Oliveira, C., Lockstone, H. E., Snellenberg, S., Grolmusova, N. and Chapman, J. R.

53BP1 Integrates DNA Repair and p53-Dependent Cell Fate Decisions via Distinct Mechanisms.

Mol Cell, (2016),

7 Fong, C. S., Mazo, G., Das, T. et al.

53BP1 and USP28 mediate p53-dependent cell cycle arrest in response to centrosome loss and prolonged mitosis.

Elife, 5 (2016),

8 Meitinger, F., Anzola, J. V., Kaulich, M. et al.

53BP1 and USP28 mediate p53 activation and G1 arrest after centrosome loss or extended mitotic duration.

J Cell Biol, 214 (2016), 155-166

$9 \quad$ Iwabuchi, K., Bartel, P. L., Li, B., Marraccino, R. and Fields, S.

Two cellular proteins that bind to wild-type but not mutant p53.

Proc Natl Acad Sci U S A, 91 (1994), 6098-6102

10 Derbyshire, D. J., Basu, B. P., Serpell, L. C. et al.

Crystal structure of human 53BP1 BRCT domains bound to p53 tumour suppressor.

EMBO J, 21 (2002), 3863-3872 
11 Joo, W. S., Jeffrey, P. D., Cantor, S. B., Finnin, M. S., Livingston, D. M. and Pavletich, N. P.

Structure of the 53BP1 BRCT region bound to $\mathrm{p53}$ and its comparison to the Brca1 BRCT structure.

Genes Dev, 16 (2002), 583-593 
117-142

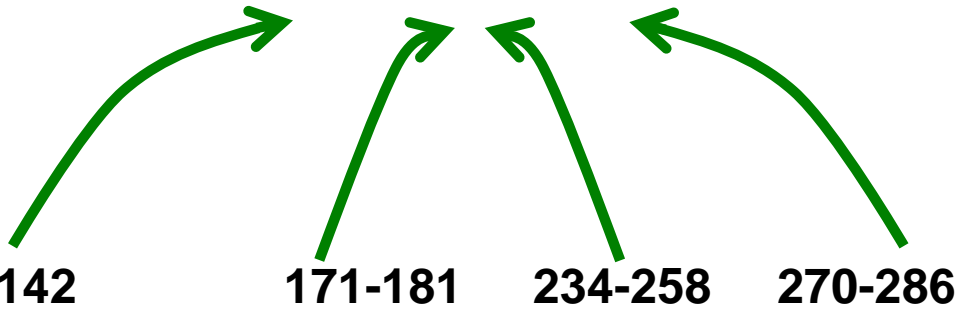

TP53

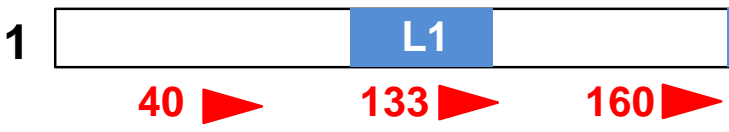

53BP1
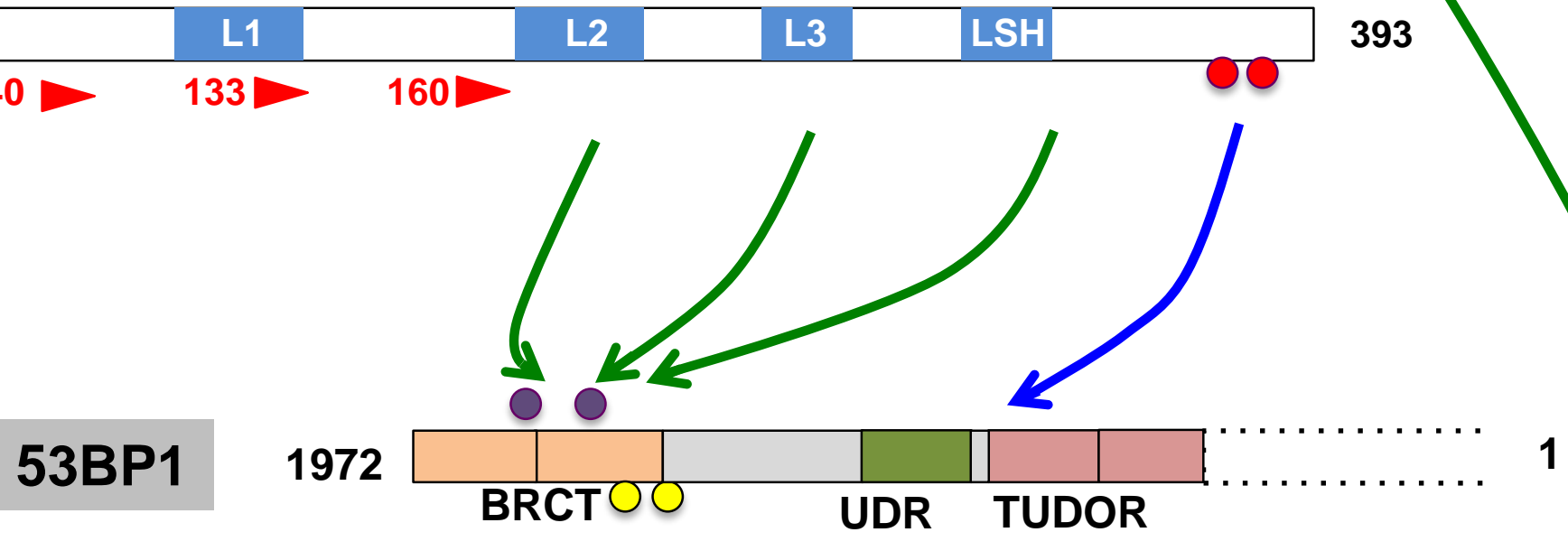

USP28

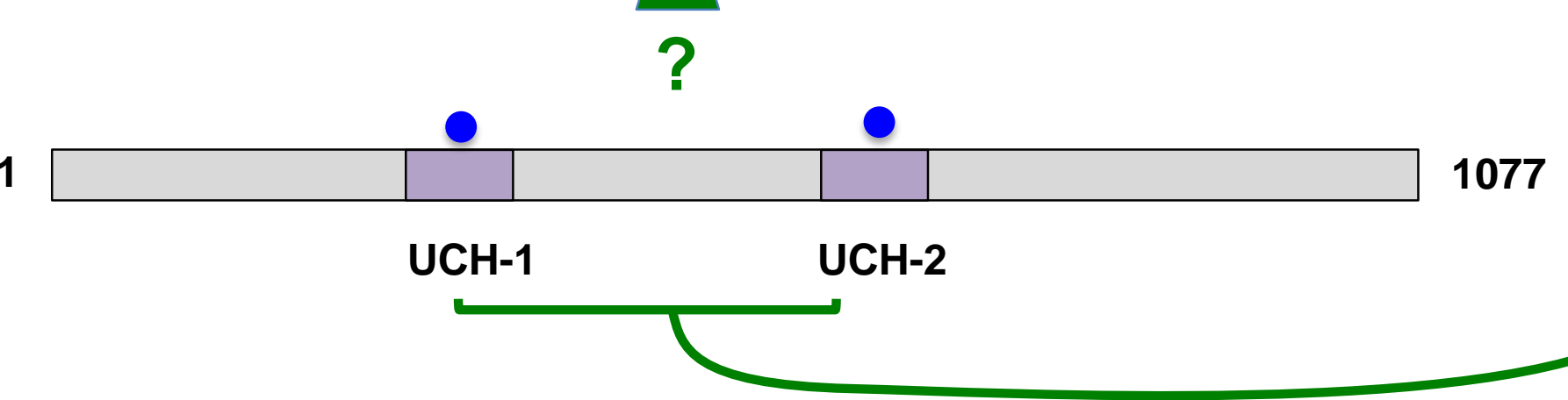

1

$\Delta$

077 